\title{
Sub-100-fs Yb:CALGO nonlinear regenerative amplifier
}

\author{
Julien Pouysegur, ${ }^{1,2, *}$ Martin Delaigue, ${ }^{2}$ Yoann Zaouter, ${ }^{2}$ Clemens Hönninger, ${ }^{2}$ Eric Mottay, ${ }^{2}$ \\ Anaël Jaffrès, ${ }^{3}$ Pascal Loiseau, ${ }^{3}$ Bruno Viana, ${ }^{3}$ Patrick Georges, ${ }^{1}$ and Frédéric Druon ${ }^{1}$ \\ ${ }^{1}$ Laboratoire Charles Fabry, Institut d'Optique, CNRS, Université Paris-Sud, 2 Ave. Augustin Fresnel, \\ Palaiseau Cedex 91127, France \\ ${ }^{2}$ Amplitude Systemes, 11 Avenue de Canteranne, Cité de la Photonique, 33600 Pessac, France \\ ${ }^{3}$ Chimie-Paristech, Laboratoire de Chimie de la Matière Condensée de Paris, CNRS-UMR 7574, \\ UPMC Univ Paris 06, 11 rue Pierre et Marie Curie, 75005 Paris, France \\ ${ }^{*}$ Corresponding author: julien.pouysegur@institutoptique.fr
}

\begin{abstract}
We report on the first diode-pumped $\mathrm{Yb}: \mathrm{CaGdAlO}_{4}$ regenerative amplifier in the sub-100-fs regime. It generates pulses at a central wavelength of $1047 \mathrm{~nm}$ with up to $24 \mu \mathrm{J}$ energy (after compression) at a repetition rate of $50 \mathrm{kHz}$. The measured pulse duration is $97 \mathrm{fs}$, with a spectral bandwidth of $19 \mathrm{~nm}$. We describe in detail how nonlinear effects are optimally used to compensate gain narrowing in order to overcome the $100 \mathrm{fs}$ barrier. (C) 2013 Optical Society of America
\end{abstract}

Many industrial applications, such as athermal micromachining [1] or eye surgery, require efficient and compact laser systems delivering ultrashort pulse energies of several tens of microjoules. To reach this energy level, femtosecond systems rely on the well-known chirped pulse amplification (CPA) concept in which nonlinear effects are minimized by sufficient temporal stretching prior to amplification. In terms of gain medium, Nd:glass bulk amplifiers have progressively been replaced by ytterbium $(\mathrm{Yb})$-doped crystalline materials that are more suited to average power scaling. Among Yb-doped materials, Yb:YAG and Yb:tungstates currently hold a dominant position. The combination of satisfying thermal conductivity together with large and broad absorption and emission cross-sections has allowed their dissemination and a new generation of ultrashort industrial amplifiers. On the other hand, the lack of crystalline disorder, responsible for the good thermal properties, also prevents the generation of sub-100-fs pulses due to the restricted available gain bandwidth. Therefore, typical durations from $\mathrm{Yb}$ :tungstate amplifiers are in the range of $200-400$ fs [2-5] while for Yb:YAG-based amplifiers, the obtained pulse widths are typically between $500 \mathrm{fs}$ and 1 ps [6,7].

Several $\bar{Y} \bar{b}$-doped crystal hosts have been studied in the past decade, mostly to generate both high average powers and shorter pulses from ultrafast oscillators. However, this work only starts to be extended to large-optical bandwidth amplifiers, among others, $\mathrm{Yb}$ : SYS [8], and more recently $\mathrm{Yb}$-doped fluorites $\mathrm{Yb}: \mathrm{CaF}_{2}$ [9-11].

The Yb:CALGO material stands out among gain media because it has the broadest emission cross-sections together with good thermal and mechanical properties [12-17]. Indeed, with a gain bandwidth of $\sim 60 \mathrm{~nm}$ spanning from 1010 to $1070 \mathrm{~nm}$, it has already been used to generate sub-50-fs pulses from ultrafast oscillators. Its thermal conductivity of $6.3-6.9 \mathrm{~W} \cdot \mathrm{K}^{-1}$ for a $2 \%$-doped matrix holds great promises with respect to average power scaling. In this Letter, we report on the first sub-100-fs regenerative amplifier based on an Yb-doped bulk gain medium. The amplifier uses a sophisticated scheme that takes advantage of nonlinearly induced spectral evolutions to counteract gain narrowing occurring during amplification [18]. With up to $24 \mu \mathrm{J}$ of output energy, this architecture is shown to hold great promises for many scientific and industrial applications.

The experimental setup is depicted in Fig. 1 and consists of an ultrafast oscillator, a negative-dispersion stretcher, an Yb:CALGO regenerative amplifier, and a compressor. The ultrafast oscillator generates a stable train of $165 \mathrm{fs}$ pulses centered at $1047 \mathrm{~nm}$ with a spectral bandwidth of $15 \mathrm{~nm}$ at $46 \mathrm{MHz}$ for an average power of 8-10 $\mathrm{mW}$. Before seeding into the regenerative amplifier, the pulses are negatively stretched to $\sim 20 \mathrm{ps}$ by use of a $800 \mathrm{l} / \mathrm{mm}$ transmission grating dispersive line. A $20 \mathrm{~mm}$ single BBO Pockels cell combined with a $2 \mathrm{~mm}$ thin film polarizer (TFP) is used as an optical gate to trap the pulse to be amplified with minimal losses inside the regenerative amplifier and extract it after amplification. The gain medium is a $4 \mathrm{~mm}$ long AR-coated $5 \% \mathrm{Yb}$-doped CALGO cut along the $a$ axis. It is pumped at $980 \mathrm{~nm}$ with a fibercoupled unpolarized laser diode delivering an average power of $12 \mathrm{~W}$. The pump beam is 1:1 imaged in the crystal through a dichroic mirror. The cavity length is $1.3 \mathrm{~m}$. A $500 \mathrm{l} / \mathrm{mm}$ grating-based compressor is used to compress

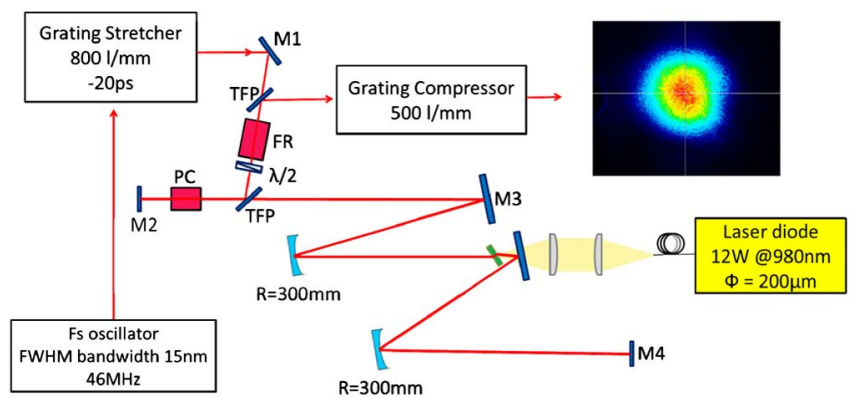

Fig. 1. Experimental setup and amplified beam profile. M, plane mirror; TFP, thin-film polarizer; PC, Pockels cell; FR, Faraday rotator. 
the output chirped pulses with an overall efficiency of $60 \%$.

In CW oscillator operation, the amplifier delivers an output average power of $3 \mathrm{~W}$ with optical-to-optical efficiency of $25 \%$. In a $Q$-switched regime, the average power is typically between $2.5 \mathrm{~W}$ at $80 \mathrm{kHz}$ and $1.1 \mathrm{~W}$ at $25 \mathrm{kHz}$. The build-up time is around $1.2 \mu \mathrm{s}$ (corresponding to $\sim 130$ roundtrips). The measured net gain per round trip is 1.09. This low gain implies a high number of roundtrips and a high sensitivity to intracavity losses. Low gain also makes the gain bandwidth more sensitive to spectrally dependent losses. The $Q$-switched spectrum (Fig. 2) is centered at $1047 \mathrm{~nm}$ and has a FWHM of about $10 \mathrm{~nm}$, slightly narrower than the seed spectrum $(15 \mathrm{~nm})$. Indeed, due to the large number of roundtrips, the gain bandwidth drops significantly due to the spectral transmission shapes of the cavity components, especially the TFP used in transmission.

The 20 ps seeded pulses exhibit a negative chirp that almost corresponds to the extra phase (dispersion) accumulated in the amplifier cavity during the build-up time. For a build-up time of $1200 \mu$ s, with a dispersion of $0.48 \mathrm{ps}^{2} / \mathrm{m}$ and $0.04 \mathrm{ps}^{2} / \mathrm{m}$ for Yb:CALGO and BBO, respectively, the total positive dispersion accumulated is approximately $0.69 \mathrm{ps}^{2}$.

The evolution of spectral bandwidth along the amplification process is shown in Fig. 2, both experimentally measured and simulated using a split-step Fourier propagation software package. During an initial phase, the pulse spectrum bandwidth slowly reduces due to gain narrowing. The pulse accumulates positive dispersion during amplification and is therefore temporally compressed. When the pulse peak power is sufficient and pulse dispersion still negative, self-phase-modulationinduced spectral compression appears [19]. Finally, in the last round trips before extraction, the pulse chirp becomes positive, and self-phase modulation then induces spectral broadening, resulting in a final bandwidth that is larger than the initial one.

In the experiment, the chirp of the input pulses is adjusted in order to generate a broad spectrum that can be compressed at the highest possible energy. The residual positive chirp is removed by the compressor and the final pulse duration revealed.

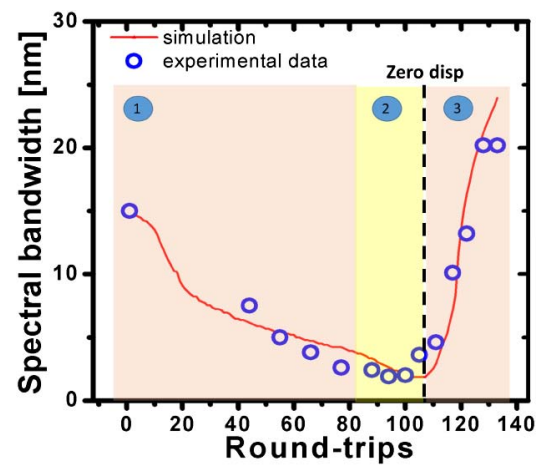

Fig. 2. Experimental data and simulation of the spectral bandwidth evolution during amplification. Region 1: amplification dominated by gain narrowing. Region 2: high peak power and negative chirp lead to spectral compression. Region 3: high peak power and positive chirp lead to spectral broadening.
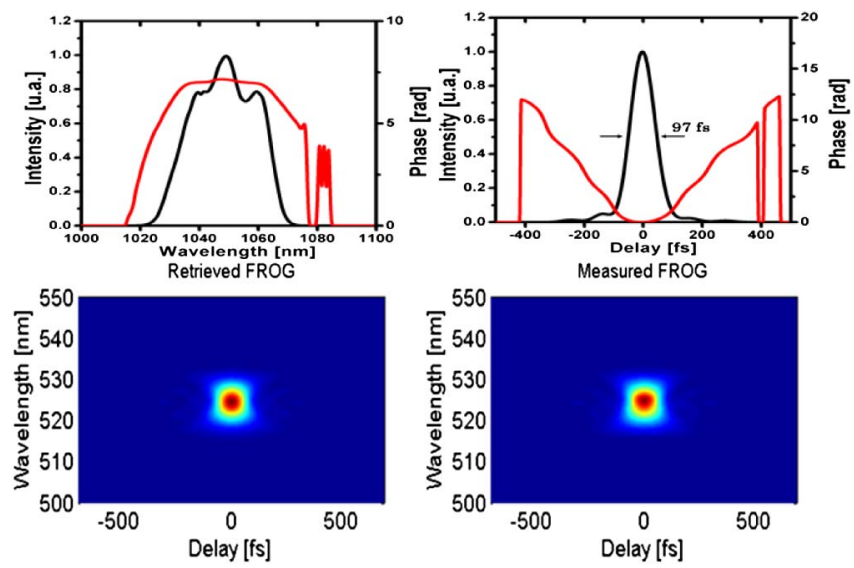

Fig. 3. Retrieved spectral and temporal profiles from SHGFROG measurement (top), and retrieved and measured FROG traces (bottom) at $50 \mathrm{kHz}$.

The shortest pulse duration is achieved at a repetition rate of $50 \mathrm{kHz}$ with $1.2 \mathrm{~W}$ of compressed average power corresponding to $24 \mu \mathrm{J}$ pulse energy. At this energy level, the autocorrelation FWHM is 3 ps before compression and $141 \mathrm{fs}$ after compression. The pulse duration and shape are characterized using a second harmonic generation frequency-resolved optical gating (SHG-FROG) setup. The results of the SHG-FROG measurement are shown in Fig. 3, and are in good agreement with independent autocorrelation and spectrum measurements. The retrieved temporal profile gives a FWHM duration of $97 \mathrm{fs}$. The pulse quality is good with a slight pedestal induced by uncompensated spectral phase. The spectrum has a FWHM of $19 \mathrm{~nm}$, corresponding to a time-bandwitdh product of 0.5. The Fourier-transform limited spectrum corresponds to a minimal pulse duration of $78 \mathrm{fs}$, indicating that further optimization of the propagation through the setup could lead to even shorter pulse durations. The compressor efficiency is limited by the optical components used, but could be optimized to provide a $25 \%$ improvement in output energy.

Selected spectra are plotted for comparison purposes in Fig. 4. The spectrum at the output of the amplifier is much broader than the spectrum obtained in $Q$-switched operation owing to the nonlinear amplification regime described above. The spectral content corresponding to the compressed pulses is reduced on its long wavelength side by the losses of the optical components of

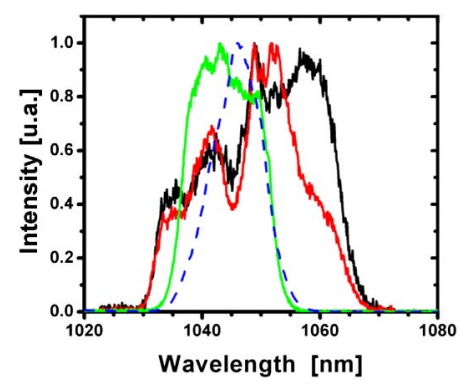

Fig. 4. Oscillator spectrum (green). Output spectrum in $Q$ switched regime (dashed blue). Regenerative amplifier output spectrum before (black) and after (red) compressor. 

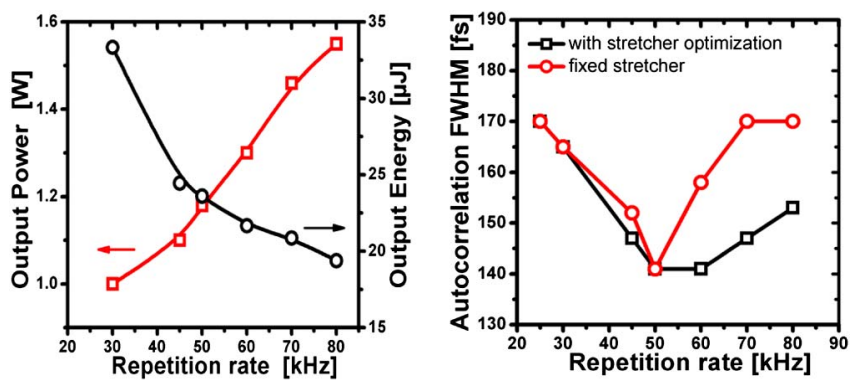

Fig. 5. (Left) Average output power (red) and pulse energy (black) as a function of repetition rate. (Right) Autocorrelation FWHM as a function of repetition rate.

the compressor. Optimization of this compressor could therefore lead to potentially shorter compressed pulses.

The spatial profile measured at the output of the regenerative amplifier is shown as an inset in Fig. 1. The output beam exhibits $M^{2}<1.2$ on both axes. Indeed, due to the spatial filtering of the regenerative amplifier cavity, spatiotemporal couplings induced by Kerr effects, which in our case are moderate per roundtrip, are essentially removed. Furthermore, for all investigated parameters, no thermal degradation of the beam profile occurs due to the large value of thermal conductivity for CALGO.

Figure 5 shows the evolution of various output parameters of the amplifier at maximum pump power as a function of the repetition rate, varying from 30 to $80 \mathrm{kHz}$ (limited by our Pockels electrical switch). Over this range, the maximum pulse energy ranges from 30 to $60 \mu \mathrm{J}$ before compression, i.e., 20 to $40 \mu \mathrm{J}$ after.

We compare two ways to optimize the system. For the first method, the stretcher is fixed for an optimal operation at $50 \mathrm{kHz}$ and only the compressor is adjusted. The pulse duration is minimized but not for optimized nonlinearity level. In this case, the pulse duration increases rapidly when the repetition rate is changed from the initial optimized operation point at $50 \mathrm{kHz}$. The other way is to optimize both stretcher and compressor to retrieve the shortest pulse duration at each repetition rate, thereby also optimizing the level of nonlinearity. This allows us to reach shorter pulses on a wider repetition rate range. In any case, the pulse duration cannot be kept short when the energy is increased too much (i.e., at repetition rates below $40 \mathrm{kHz}$ ) due to excessive and uncompressible accumulated nonlinear phase. For repetition rates above $70 \mathrm{kHz}$, the energy per pulse is not sufficient to create enough bandwidth, also leading to longer pulse widths.

Figure $\underline{6}$ shows different autocorrelations acquired at 25,50 , and $80 \mathrm{kHz}$. At the lowest repetition rate, the duration cannot be kept short as uncompressible phase terms are generated. This remaining phase also creates a growing pedestal. At $80 \mathrm{kHz}$, the autocorrelation quality is close to that obtained at $50 \mathrm{kHz}$. Only the duration is slightly longer. Still, the variation of duration is kept below $20 \%$, with a maximum pulse duration of $<120 \mathrm{fs}$, between 25 and $80 \mathrm{kHz}$, and is therefore acceptable for most applications in which this type of laser would be useful.

The sub-100-fs regime appears at a well-defined energy, or equivalently repetition rate range, and the cavity
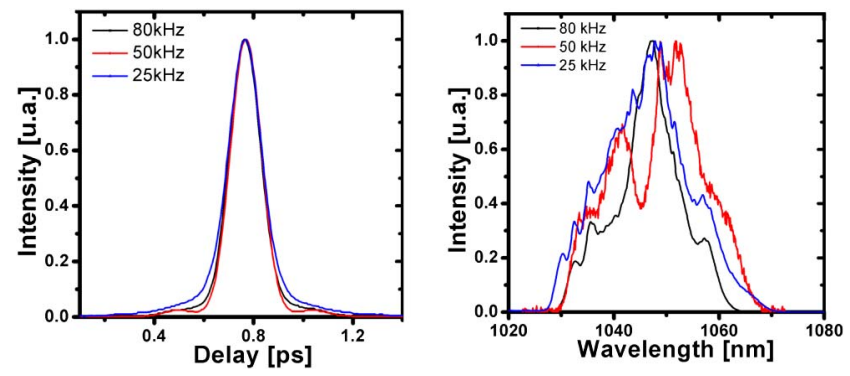

Fig. 6. Autocorrelation (left) and spectrum (right) for different repetition rates: $80 \mathrm{kHz}$ (black), $50 \mathrm{kHz}$ (red), and $25 \mathrm{kHz}$ (blue).

parameters such as pump power, beam waist in the BBO, and $\mathrm{Yb}: \mathrm{CALGO}$ have to be adjusted to fit the desired pulse energy.

To conclude, we demonstrate, to the best of our knowledge, the first sub-100 fs regenerative bulk amplifier based on an $\mathrm{Yb}$-doped material. In our experiment, the combination of a broad gain bandwidth gain medium such as Yb:CALGO together with an innovative strategy of gain narrowing mitigation allowed the generation of $24 \mu \mathrm{J} 97 \mathrm{fs}$ pulses leading to a peak power of $218 \mathrm{MW}$ with up to $50 \mathrm{~dB}$ of accumulated gain. For the optimum operation point, the pulse evolution is first dominated by spectral compression before spectral broadening occurs during the last roundtrips. An increased pulse energy of $55 \mu \mathrm{J}$ before compression ( $32 \mu \mathrm{J}$ after) has also been obtained, while retaining good temporal quality with 120 fs pulses. Using more adapted compressor components is an easy way to further increase the energy. The main drawback of this technique lies in the restricted energy range for which short pulses with good temporal quality can be generated. Because of its thermal and spectroscopic properties, we believe that Yb:CALGO regenerative amplifiers can be scaled to multiple tens of Watt with short pulse duration. Further reduction of the pulse duration could be achieved by optimizing the stretcher-compressor design and the spectrally dependent losses in the regenerative amplifier cavity. We also believe that such sources will find many applications in the scientific and industrial communities.

The work described here has been partly funded by the French National Research Agency (ANR) through the Femtocryble program.

\section{References}

1. S. Nolte, C. Momma, H. Jacobs, A. Tünnermann, B. N. Chichkov, B. Wellegehausen, and H. Welling, J. Opt. Soc. Am. B 14, 2716 (1997).

2. M. Larionov, F. Butze, D. Nickel, and A. Giesen, Opt. Lett. 32, 494 (2007).

3. M. Delaigue, I. Manek-Hoenninger, F. Salin, C. Hoenninger, P. Rigail, A. Courjaud, and E. Mottay, Appl. Phys. B 84, 375 (2006).

4. U. Buenting, H. Sayinc, D. Wandt, U. Morgner, and D. Kracht, Opt. Express 17, 8046 (2009).

5. G. H. Kim, J. H. Yang, D. S. Lee, A. V. Kulik, E. G. Sall', S. A. Chizhov, U. Kang, and V. E. Yashin, J. Opt. Technol. 80, 142 (2013).

6. C. Hönninger, I. Johannsen, M. Moser, G. Zhang, A. Giesen, and U. Keller, Appl. Phys. B 65, 423 (1997).

7. K. Sueda, S. Kawato, and T. Kobayashi, Laser Phys. Lett. 5, 271 (2008). 
8. P. Raybaut, F. Druon, F. Balembois, P. Georges, R. Gaumé, B. Viana, and D. Vivien, Opt. Lett. 28, 2195 (2003).

9. S. Ricaud, F. Druon, D. N. Papadopoulos, P. Camy, J. Doualan, R. Moncorgé, M. Delaigue, Y. Zaouter, A. Courjaud, P. Georges, and E. Mottay, Opt. Lett. 35, 2415 (2010).

10. A. Pugžlys, G. Andriukaitis, A. Baltuška, L. Su, J. Xu, H. Li, R. Li, W. J. Lai, P. B. Phua, A. Marcinkevičius, M. E. Fermann, L. Giniūnas, R. Danielius, and S. Ališauskas, Opt. Lett. 34, 2075 (2009).

11. S. Ricaud, P. Georges, P. Camy, J.-L. Doualan, R. Moncorgé, A. Courjaud, E. Mottay, and F. Druon, Appl. Phys. B 106, 823, (2012).

12. A. Agnesi, A. Greborio, F. Pirzio, G. Reali, J. Aus der Au, and A. Guandalini, Opt. Express 20, 10077 (2012).

13. E. Caracciolo, M. Kemnitzer, A. Guandalini, F. Pirzio, J. Aus der Au, and A. Agnesi, Opt. Lett. 38, 4131 (2013).
14. S. Ricaud, A. Jaffres, K. Wentsch, A. Suganuma, B. Viana, P. Loiseau, B. Weichelt, M. Abdou-Ahmed, A. Voss, T. Graf, D. Rytz, C. Hönninger, E. Mottay, P. Georges, and F. Druon, Opt. Lett. 37, 3984 (2012).

15. A. Greborio, A. Guandalini, and J. Aus der Au, Proc. SPIE 8235, 823511 (2012).

16. Y. Zaouter, J. Didierjean, F. Balembois, G. L. Leclin, F. Druon, P. Georges, J. Petit, P. Goldner, and B. Viana, Opt. Lett. 31, 119 (2006).

17. F. Druon, M. Olivier, A. Jaffrès, P. Loiseau, N. Aubry, J. DidierJean, F. Balembois, B. Viana, and P. Georges, Opt. Lett. 38, 4138 (2013).

18. P. Raybaut, F. Balembois, F. Druon, and P. Georges, IEEE J. Quantum Electron. 41, 415 (2005)

19. S. A. Planas, N. L. Pires Mansur, C. H. B. Cruz, and H. L. Fragnito, Opt. Lett. 18, 699 (1993). 